\title{
A UTILIZAÇÃO DO SISTEMA DE PREPARAÇÃO DE TEXTOS LATEX NA PRODUÇÃO DE TEXTOS ACADÊMICOS NO BRASIL: UMA INVESTIGAÇÃO PRELIMINAR E PERSPECTIVAS
}

\section{USAGE OF LATEX FOR TYPESETTING SCHOLARLY TEXTS IN BRAZIL: A PRELIMINARY STUDY AND FUTURE PERSPECTIVES}

\begin{abstract}
Youssef Alvarenga Cherem - ycherem@gmail.com Doutorado em Antropologia Social pela Universidade Estadual de Campinas (UNICAMP). Professor do Departamento de História da Arte da Universidade Federal de São Paulo (UNIFESP).
\end{abstract}

\section{RESUMO}

Introdução: LaTeX é um sistema de preparação eletrônica de textos baseado no sistema tipográfico TeX. Neste artigo, apontamos os principais benefícios e desafios de utilização dessa ferramenta, bem como perspectivas para utilização a curto e médio prazo no Brasil.

Objetivo: analisar o uso do software LaTeX na produção de textos acadêmicos no Brasil.

Metodologia: Revisão bibliográfica e análise documental.

Resultados: A utilização de uma linguagem de mark-up como LaTeX pode trazer benefícios tanto do ponto de vista do produto final e sua relação com o leitor, quanto do ponto de vista do processo de edição do texto. Apesar disso, seu uso no Brasil é bastante restrito.

Conclusões: Um dos maiores desafios para a disseminação de seu uso é a falta de apoio institucional oficial na área acadêmica e científica. Sua aceitação institucional, bem como iniciativas de capacitação do usuário, são apontados como fatores que podem mudar esse quadro.

Palavras-chave: Design gráfico. Tipografia. Design da informação. Comunicação científica. 


\section{INTRODUÇÃO}

O "design da informação" pode ser definido como um campo de estudos interdisciplinar, que relaciona tipografia, design gráfico, linguística, psicologia, ergonomia e computação, entre outras disciplinas (SOCIETY FOR TECHNICAL COMMUNICATION, 2005). Segundo a Sociedade Brasileira de Design da Informação (SBDI), um dos objetivos fundamentais do design da informação é "otimizar o processo de aquisição da informação através de matérias gráficos, sejam eles analógicos ou digitais." (SOCIEDADE BRASILEIRA DE DESIGN DA INFORMAÇÃO, 2014). Se o design da informação diz respeito a dar forma e apresentar o conteúdo de uma mensagem, adaptando-a ao seu meio de comunicação e ao seu público (ver várias definições no site do International Institute for Information Design (IIID): http://www.iiid.net/Information.aspx), podemos argumentar que sua raiz já estava presente em desenvolvimentos da forma escrita desde a antiguidade. O recurso a marcadores visuais para estruturar o texto já era discernível em manuscritos antigos, desde a introdução da pontuação na antiguidade (PUNCTUATION, 2011) e da separação das palavras nos manuscritos medievais (SAENGER, 2000), até a disposição do texto em manuscritos medievais europeus (GUMBERT, 1993) ou islâmicos (BLAIR, 2006). De fato, a aparência dos primeiros livros impressos na Europa no século XV procurava deliberadamente imitar a forma dos manuscritos. Finalmente, o sistema de pontuação e divisão por parágrafos, paginação, índices e capítulos empregado atualmente tomou forma por volta do século XVI (CHAPPELL, 1970), época em que também foi criado o tipo itálico, baseado na caligrafia chanceleresca italiana, completando assim a divisão entre,, de um lado, tipos romanos e itálicos, e, de outro, os tipos "góticos" (Textura, Fraktur, Schwabacher) utilizados principalmente em países de língua germânica (DODD, 2006; BAIN e SHAW, 1998). Desta forma, na linha de Bringhurst (2013), Unger (2007) e Tschichold (2007), inter alia, podemos afirmar, referindo-nos aos princípios estéticos que guiam a prática da palavra impressa, que houve apenas "uma mudança marginal na tipografia durante mais de cinco séculos." (UNGER, 2007, p. 14). A ideia subjacente a nossa abordagem é que, portanto, são válidos tanto para a apresentação de livros quanto de periódicos os mesmos princípios de organização da palavra escrita através da composição tipográfica e a inter-relação 
entre os elementos gráficos (figurativos) e tipográficos do texto.

Neste artigo, procurar-se-á mostrar que a utilização do software LaTeX de modo condizente com a tradição tipográfica pode auxiliar e facilitar a comunicação escrita, especialmente no caso de textos científicos ou acadêmicos. São feitas referências aos princípios e práticas tratados por Bringhurst (2013), Tschichold (2007), Ruder (1977), Felici (2012), Tracy (2003), Pettersson (2012) e Lupton (2004), especialmente no que tange a questões de legibilidade, princípios estéticos como harmonia e proporção, qualidade, custos e acesso à informação. Em relação ao resultado físico (impresso) ou eletrônico, o uso do LaTeX facilita ao leitor a visualização de dados, tanto numa leitura preliminar quanto imersiva, ajudando-o a percorrer o texto e encontrar informação com mais agilidade. De outro ponto de vista, ajuda o autor ou redator a estruturar, dispor e apresentar a informação de modo a facilitar a leitura.

\section{O SISTEMA LaTeX E A TRADIÇÃO TIPOGRÁFICA}

LaTeX, criado em 1984 por Leslie Lamport (LAMPORT, 1994), é um sistema de preparação de documentos e uma linguagem de marcação (markup language) baseado no sistema tipográfico TeX, criado por Donald Knuth em 1978 (KNUTH, 1984). Ao descrever o modus operandi de seu sistema, Lamport faz uma comparação com a tipografia antes do advento do computador, em que o trabalho era dividido entre o autor do manuscrito, o designer, e o tipógrafo. Assim, ele afirma: "LaTeX é teu designer tipográfico, e TeX é teu tipógrafo" (LAMPORT, 1994, p. 6). A abordagem do LaTeX é lógica, traduzindo visualmente, segundo parâmetros predefinidos, comandos que descrevem a estrutura lógica do texto.

Devido à sua abordagem não-visual e baseada em comandos que são essencialmente texto puro ("não formatado"), o LaTeX é contrastado com "processadores de texto" (como Microsoft Word) ou programas de diagramação de texto (como InDesign). Especialmente no caso dos processadores de texto, não fica clara a divisão entre o autor e o designer: o autor do texto, a não ser que utilize um modelo (template) prévio, é forçado a explicitar de forma visual (uma série de cliques com o mouse) suas escolhas em relação à apresentação do texto, ou então forçado a implementar um padrão tipográfico a partir de uma norma geral. O propósito do 
LaTeX é proporcionar aos autores a melhor qualidade tipográfica possível, com um mínimo de esforço ou de conhecimento técnico: "LaTeX foi concebido para livrar-te de preocupações em relação à formatação, permitindo que te concentres na escrita. Se gastas muito tempo a te preocupares com a forma, estás a utilizar incorretamente o LaTeX" (LAMPORT, 1994, p. 8). Em outras palavras, uma vez já definido o aspecto visual final da publicação - seja por um designer, um programador, ou uma combinação de ambos -, o processo de edição do texto suprime a figura do tipógrafo e do designer, que serão cumpridas pelo programa (LaTeX). Essa separação entre forma e conteúdo é especialmente útil ao concentrar a atenção do redator ou autor no conteúdo do texto, e não em sua aparência final. O próprio processo de trabalho para produção de um texto utilizando LaTeX impõe uma separação visual entre o arquivo-fonte e seu resultado imprimível. O usuário deve empregar comandos que explicitem a estruturação lógica do texto (partes, capítulos, seções etc.).

Segundo Emil Ruder, "a exigência mais urgente que se deve fazer à tipografia é dividir e organizar elementos diversos." (RUDER, 1977, p. 14). A linguagem de mark-up empregada no LaTeX permite aos autores, através de comandos textuais relativamente simples, inserir - e, ao mesmo tempo, formatar - através de escolhas previamente definidas e externas ao texto propriamente dito - todos os elementos necessários para dar forma ao texto final, pronto para impressão. Há um sistema para criação de bibliografias (CHEREM, 2013, p. 25-36; Van DONGEN, 2012, p. 2529; GOOSENS; SAMARIM; MITTELBACH, 1994, p. 371-419; LEHMAN et al., 2013;) e índices (Van DONGEN, 2012, p. 30-32; OETIKER et al., 2011, p. 86-87; GOOSENS; SAMARIM; MITTELBACH, 1994, p. 345-356). Obtêm-se facilmente referências cruzadas (OETIKER et al., 2011, p. 42); inserção de figuras, tabelas e legendas, com suas respectivas listas de tabelas e de figuras no sumário (CHEREM, 2013, p. 37-39, 61-69; Van DONGEN, 2012, p. 75-79; OETIKER et al., 2011, p. 4950). Elementos matemáticos como equações, matrizes, símbolos e teoremas também têm tratamento extremamente eficiente (GRATZER, 2000; OETIKER et al., 2011, p. 53-82). Todos esses elementos são numerados automaticamente e, se necessário, referenciados através de "rótulos" criados pelo próprio usuário.

LaTeX é especialmente útil para gerar resultados ótimos em termos de textos complexos, principalmente textos científicos onde há muitas fórmulas e/ou símbolos. 
A utilização do sistema de preparação de textos LaTeX na produção de textos acadêmicos no Brasil: uma investigação preliminar e perspectivas

Mas suas vantagens não se limitam a expressões matemáticas, estendendo-se também para fatores que influenciam a legibilidade do texto.

O vocabulário técnico inglês distingue entre readability e legibility. Readability é conforto para ler textos por períodos extensos de tempo, enquanto que legibility é a capacidade de reconhecer e distinguir caracteres isolados (TRACY, 2003, p. 3031). Em português, poderíamos distinguir entre a legibilidade do texto e a legibilidade da fonte. É bom notar que o debate na literatura especializada sobre a legibilidade (do texto e dos caracteres) em fontes com ou sem serifas não permite uma decisão categórica entre uma ou outra (POOLE, 2008), apesar de tradicionalmente se afirmar que fontes serifadas facilitariam a leitura imersiva, de textos longos, devido à ênfase horizontal criada pelas serifas. Também não se pode deixar de notar que a escolha da fonte também deve ser adaptada ao seu meio de reprodução (tela, tipo de papel, cor, tamanho, tipo de impressão, etc.). Outro fator ser considerado é a afinidade cultural ou contato com determinado tipo ${ }^{1}$ Assim, há vários argumentos sobre e superioridade dos tipos chamados "Fraktur", amplamente utilizados na Alemanha até meados do século $\mathrm{XX}$, especialmente em relação à adaptação à língua alemã, à economia de espaço e à facilidade de leitura, embora contemporaneamente esses tipos sejam considerados muito pouco legíveis pelo leitor comum (BAIN; SHAW, 1998).

Para otimizar a legibilidade do texto, em ambos os seus aspectos (readability e legibility), LaTeX lida automaticamente com: kerning (espaçamento entre pares de letras); versaletes verdadeiros (ao invés de versaletes "falsos", em que é simplesmente ajustado o tamanho das letras); fonte adaptada ao tamanho (optical sizes), quando disponível [NOTA: Para aumentar a legibilidade de caracteres pequenos ( 6 a 8 pontos), ou salientar a elegância de tipos maiores (24 pontos e acima $)^{2}$. É interessante notar que uma das primeiras fontes digitais a utilizar de tamanhos óticos é a família Computer Modern, de Knuth, a fonte default em LaTeX.]; ligaduras comuns (evitando choque entre pares ou conjuntos de letras como fi, ff etc.) ou históricas; e quebras de linha, hifenização e justificação. Em muitos processadores de texto, ou mesmo programas para diagramação eletrônica (desktop

\footnotetext{
1 Aqui utilizamos o termo "tipo" (typeface) para designar os caracteres de imprensa. A palavra "fonte", embora usualmente empregada no mesmo sentido, mais especificamente uma espécie de um determinado tipo gráfico; assim, por exemplo, Times New Roman regular, tamanho 12, é uma fonte, enquanto que Times New Roman é um tipo.

${ }^{2}$ Ver exemplos em: https://www.adobe.com/type/topics/opticalsize.html.
} 
publishing, ou DTP), e principalmente no caso de textos justificados se não houver uma intervenção ativa do designer parágrafo por parágrafo (ou, em alguns casos, linha por linha), pode ser gerado um espaçamento muito curto ou muito longo entre as letras e palavras. O espaçamento inadequado afeta a negativamente a legibilidade dos caracteres ao tornar grupos de caracteres ambíguos (FELICl, 2012, p. 108) e prejudicando a legibilidade ao romper a harmonia do bloco de texto (FELICl, 2012, p. 163-164).

Para otimizar a legibilidade, uma linha não pode ser tão longa que dificulte o caminho que o olho do leitor percorre do final uma linha ao começo de outra (FELICI, 2012, p. 110), recomendando-se algo em torno de 60 caracteres por linha (RUDER, 1977, p. 14). O espaçamento entre linhas (leading) não pode ser tão estreito que confunda a leitura, nem tão largo a ponto de romper o padrão do texto. O sistema LaTeX lida com todos esses aspectos - da divisão e organização dos elementos do texto, passando pela legibilidade, extensão da linha e justificação.

\section{BENEFÍ́CIOS E PARTICULARIDADES DO USO DE LaTeX NO FLUXO DE TRABALHO EDITORIAL}

A utilização do LaTeX também implica outros benefícios. Trata-se de um programa gratuito e de código aberto (open source). Os custos explícitos e implícitos (segurança, manutenção) de um software não devem ser subestimados. ${ }^{3}$ Embora recentemente tenham começado a surgir descontos para estudantes, instituições de ensino e organizações com fins não lucrativos, os procedimentos burocráticos tanto da parte da empresa vendedora do software quanto da parte da organização, empresa ou órgão da administração pública que irão utilizá-lo - são um sério obstáculo a ser superado. Em alguns casos, como para instituições de ensino que não dispõem de financiamento suficiente, alternativas gratuitas podem diminuir de forma significativa o custo de seu material, com um custo de implementação e manutenção de soluções em TI se tornando extremamente reduzido ou até mesmo

3 Uma assinatura anual do Microsoft Office para uso doméstico (5 usuários) custa cerca de $R \$ 200$. Há desconto educacional, mas os procedimentos e a burocracia são impeditivos. Uma assinatura completa da Creative Cloud, da Adobe, custa $\mathrm{R} \$ 109$ ao mês, no plano anual (https://creative.adobe.com/plans? plan=individual\&store_code=br). O InDesign sem assinatura custa 1.169 dólares, e a Creative Suite Design Standard (Photoshop, Illustrator, InDesign, Acrobat $X$ Pro, Bridge e Media Encoder) custa 2.525 dólares. 
A utilização do sistema de preparação de textos LaTeX na produção de textos acadêmicos no Brasil: uma investigação preliminar e perspectivas

insignificante. No Brasil, o Governo Federal tem fomentado o uso de software livre. ${ }^{4}$

$\mathrm{O}$ fato de ser um programa de código aberto significa que o usuário tem a liberdade de: a) utilizar o programa para qualquer propósito; b) estudar o funcionamento do programa e mudá-lo para atender a suas necessidades; c) criar versões modificadas ou derivadas do programa; d) redistribuir cópias (THE FREE SOFTWARE FOUNDATION, 2014)5.). Além disso, TeX/LaTeX é um programa estável. Um arquivo salvo há 30 anos no formato tex pode ser aberto, editado e compilado exatamente da mesma forma hoje. É também um formato portátil e multiplataforma, podendo ser utilizado e instalado na maioria dos sistemas operacionais mais comuns - UNIX, Mac, Windows, Linux, Free BSD etc. Sua execução é extremamente econômica em termos de memória e processamento; equipamentos com mais de uma década podem facilmente processar documentos de centenas de páginas. Em relação a textos técnicos ou científicos, o LaTeX é um dos formatos padrão para periódicos e publicações de áreas como matemática, física, engenharia e computação, sendo utilizado e difundido por instituições como o Institute for Electrical and Electronics Engineers (IEEE), a American Mathematical Society (AMS), e editoras como Elsevier e Springer. Finalmente, o LaTeX tem uma grande e bastante ativa comunidade de usuários dispostos a desenvolver soluções, e ajudando os usuários desde as dúvidas triviais até as mais complexas. ${ }^{6}$

Além dos custos relacionados ao próprio software, os custos do processo de produção de material científico impresso ou eletrônico são altíssimos. Revistas internacionais de ponta de áreas de ciências exatas, médicas ou biológicas têm custos maiores, ligados também à grande taxa de recusa dos artigos submetidos. King (2007), apresentando dados de estudos de periódicos de várias áreas, mostra que o custo por artigo publicado pode variar desde poucas centenas até milhares de dólares. O custo médio de diagramação é também é muito variável, podendo ir de cerca de USD 100 a USD 1.840 (KING, 2007, p. 97). O uso de LaTeX diminui substancialmente tanto o tempo necessário para a diagramação quanto os custos de diagramação para a editora. De fato, embora haja tendência a variação dependendo

\footnotetext{
4 Ver, entre outros, "Guia Livre: Referência de Migração para Software Livre do Governo Federal": http://www.governoeletronico.gov.br/anexos/guia-livre-versao-1.0; e o Portal do Software Livre: http://www.softwarelivre.gov.br/. É também afirmado que "O software livre deve ser entendido como opção tecnológica do governo federal" (http://www.governoeletronico.gov.br/o-gov.br/principios).

${ }^{5}$ Ver também definição em: The Open Source Initiative (2014).

6 Ver, entre outros, o fórum tex.stackexchange.com.
} 
da complexidade tipográfica do texto (diretamente proporcional à quantidade $\mathrm{e}$ complexidade de equações, fórmulas etc., figuras e gráficos), o tempo médio para revisar elementos como equações, figuras, tabelas, índices etc. é muito inferior relativamente ao fluxo de trabalho (do autor e do diagramador/tipógrafo) usual, em que há de fato duas formatações do texto: uma para edição, outra para publicação ou impressão. O aspecto do artigo enviado para avaliação é completamente modificado. O trabalho do autor para "formatar" seu texto de acordo com as normas de uma publicação é cabalmente desfeito pelo diagramador, o que faz sua formatação ser útil, somente, talvez, para o revisor do artigo. No entanto, tem sido prática recorrente prescindir de um trabalho tipográfico especializado principalmente depois do advento da internet e do desktop publishing, e especialmente no caso de revistas acadêmicas e científicas de pequeno porte. Podemos perceber claramente, inclusive, em vários periódicos com versão eletrônica, que o trabalho de diagramação não é feito por um profissional. No caso do LaTeX, o layout do artigo que o autor submete à avaliação de um periódico é o mesmo que será entregue para publicação. Não é necessário o acompanhamento constante de um programador depois de definido o layout da publicação, mas mesmo assim poderemos assegurar uma qualidade superior de composição de página. As figuras 1 e 2 comparam o mesmo artigo tipografado em LaTeX e Word. $\mathrm{O}$ mesmo texto ocupa a extensão de 18 páginas, no exemplar original, e 10 páginas, na composição em LaTeX: uma economia de 44\%. Outros aspectos divergentes podem ser notados: o artigo original não tem hifenização; utiliza fontes banais e que não coadunam com o tema da publicação, e combina fontes inadequadamente.

Dois dos principais pontos negativos apontados para um uso mais amplo do LaTeX, e argumentos contra sua utilização, são a dificuldade de aprendizado e a dificuldade de personalização. No entanto, a falta de familiaridade com um tipo de ferramenta informatizada para gerar certo resultado prático ou concreto é o primeiro impedimento à sua utilização. A maioria dos usuários de processadores de texto (Word ou LibreOffice) somente faz uso sumário de sua potencialidade. As próprias regras de redação de várias instituições acadêmicas, organizações, repartições públicas ou publicações, embora às vezes bastante detalhadas, muitas vezes não passam de uma padronização arbitrária, que frequentemente impõe regras tipográficas rebuscadas, excessivas e confusas, que, além de, em muitos casos, não 
A utilização do sistema de preparação de textos LaTeX na produção de textos acadêmicos no Brasil: uma investigação preliminar e perspectivas

beneficiarem a legibilidade do texto, tornam o processo de redação segundo essas normas um verdadeiro estorvo. O apego desnecessário à "microgestão tipográfica" e o esforço por respeitar e fazer cumprir requisitos formais supérfluos pode ter como consequência a mistificação do trabalho acadêmico, incluindo-se a confusão frequente de metodologia científica, redação acadêmica, e normas da Associação Brasileira de Normas Técnicas (ABNT). ${ }^{7}$

$7 \mathrm{Um}$ exemplo da excessiva regulamentação é a quantidade de normas criadas pela ABNT para textos acadêmicos. Existe uma norma para sumários (NBR 6027), uma para resumos (NBR 6028), uma para índices (NBR 6034), uma para apresentação de trabalhos acadêmicos (NBR 14724) e outra para numeração de seções de documentos (NBR 6024). Essas normas, com influência nacional, são editadas e modificadas sem nenhum benefício palpável para a organização da informação do texto técnico, acadêmico ou científico. A situação se torna mais intrigante ao darmos conta de que as normas NBR da ABNT não são vinculantes (obrigatórias), segundo os próprios termos da Associação: "ABNT NBR é a sigla de Norma Brasileira aprovada pela ABNT, de caráter voluntário, e fundamentada no consenso da sociedade. Torna-se obrigatória quando essa condição é estabelecida pelo poder público." (http://abnt.org.br/paginampe/perguntas-e-respostas, grifo nosso). A esse caráter voluntário da adoção das normas de redação científica preconizadas por essa instituição - para não mencionar ainda a presunção de consenso (aceitação tácita de seus pressupostos e conclusões) dos grupos sociais às quais tais normas dizem respeito - soma-se a obrigatoriedade do cumprimento de interpretações dessa norma, em que o "recomendado" se torna "obrigatório", e em que o que era facultativo não mais o é. A "norma" ABNT, em abstrato, a cujos originais seus agentes implementadores às vezes nem têm acesso, é "reciclada" em vários níveis, da universidade, aos institutos, departamentos, bibliotecas, orientadores, secretárias e bibliotecários. Isso quando a norma é aceita - e existem casos em que isso não acontece, sem nenhum efeito nocivo à produção científica. 
Youssef Alvarenga Cherem

A utilização do sistema de preparação de textos LaTeX na produção de textos acadêmicos no Brasil: uma investigação preliminar e perspectivas

Figura 1 - Exemplo de artigo acadêmico composto em Microsoft Word.

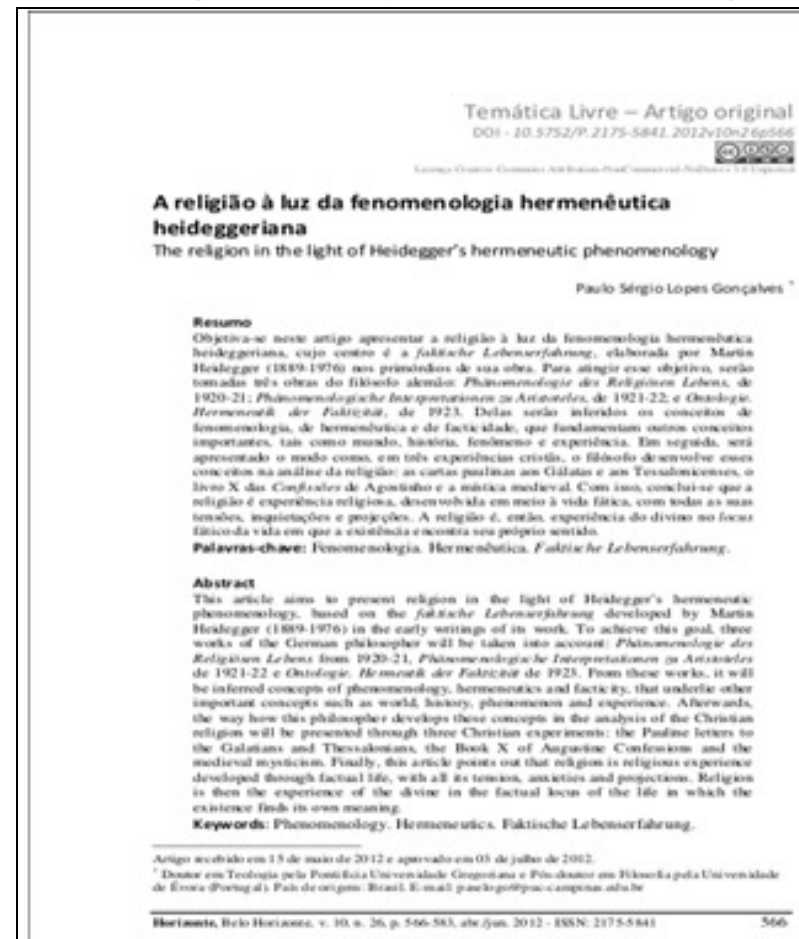

(a)

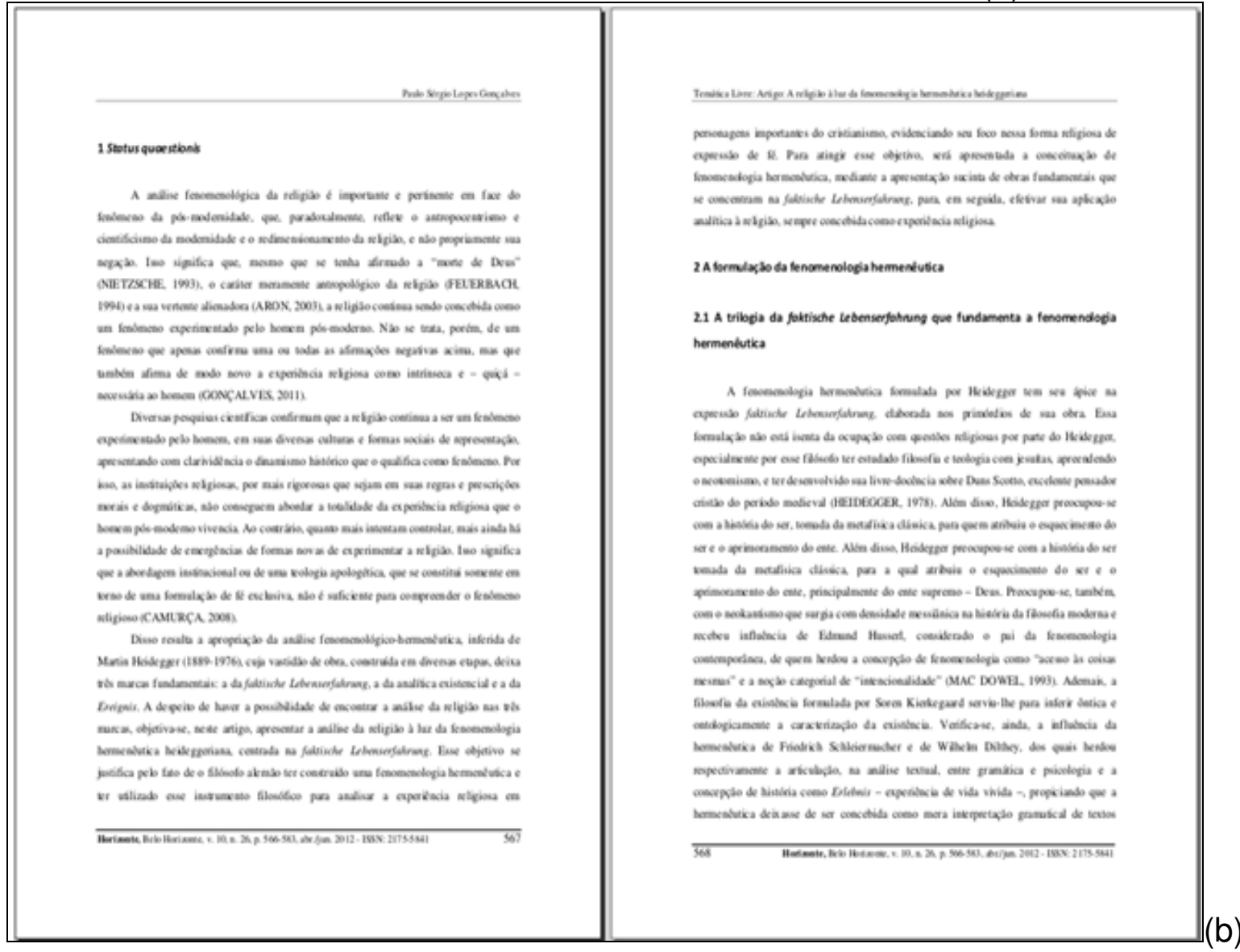

Fonte: http://periodicos.pucminas.br/index.php/horizonte/article/view/P.2175-5841.2012v10n26p566

Inf. Inf., Londrina, v. 20, n. 1, p. 228 - 249, jan./abr. 2015. 
Youssef Alvarenga Cherem

A utilização do sistema de preparação de textos LaTeX na produção de textos acadêmicos no Brasil: uma investigação preliminar e perspectivas

Figura 2 - Exemplo do artigo da figura 1, composto em LaTeX. Foi modificada a paginação para abertura em página ímpar.

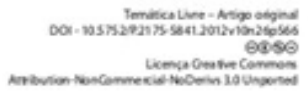

A religião à luz da fenomenologia hermenêutica heideggeriana

The religion in the light of Heidegper's hermenevik phenomenoiogy

Pudo Strge Lops Geapulves'

2012

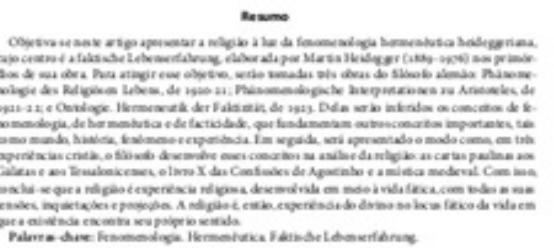

Abasant

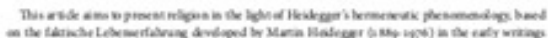

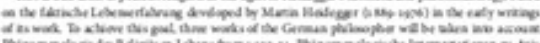

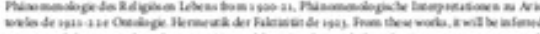

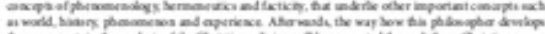

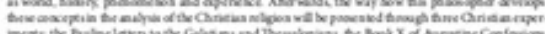

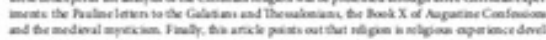

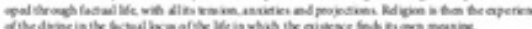

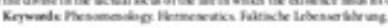
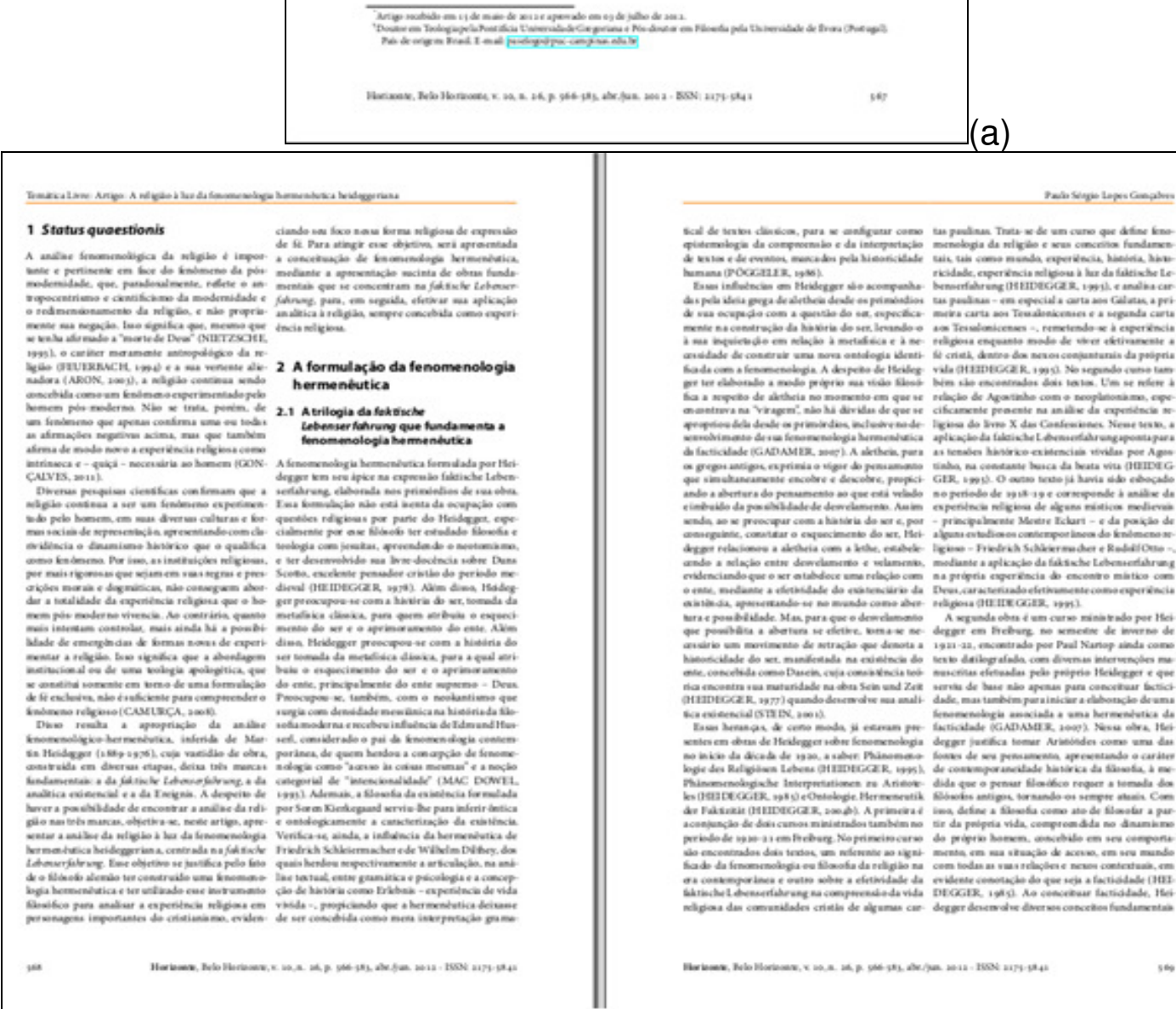

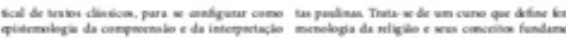

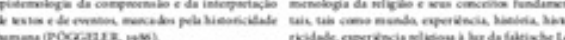

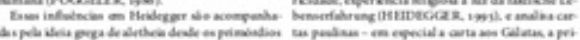

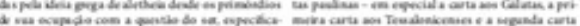

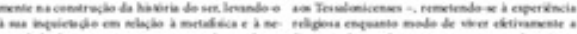

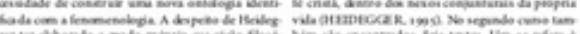

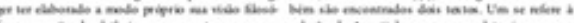

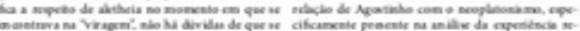

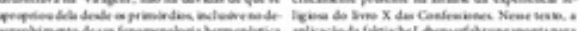

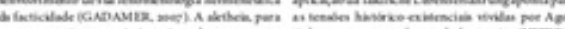

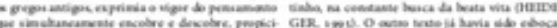

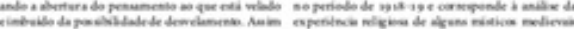

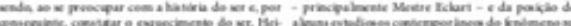

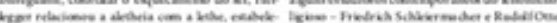

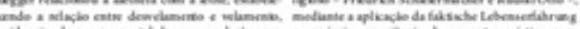

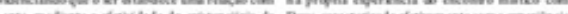

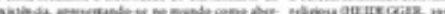

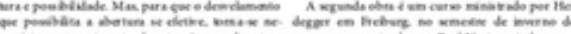

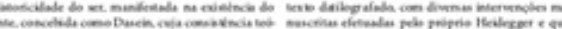

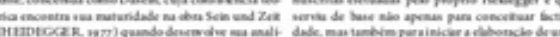

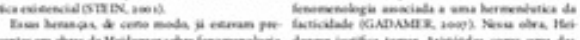

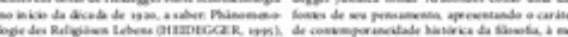

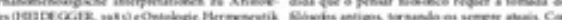

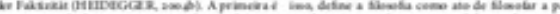

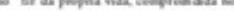

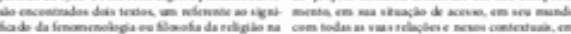

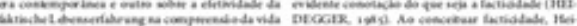

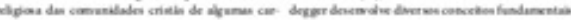

Fonte: $\mathrm{O}$ autor. 
A utilização do sistema de preparação de textos LaTeX na produção de textos acadêmicos no Brasil: uma investigação preliminar e perspectivas

Outro ponto negativo do LaTeX frequentemente apontado seria a difícil personalização dos documentos. Como o LaTeX é um conjunto de macros, a definição da aparência final é realizada modificando as macros utilizadas ou criando novas macros. Existe aí uma relativa especialização de funções entre o escritor, o designer e o programador. Este último implementa as opções gráficas do designer; ao escritor competiria, em última análise, somente a redação do texto. Nesse sentido, grande parte das "escolhas" que um autor poderia realizar é estabelecida de antemão pelo que se denomina uma "classe" na terminologia LaTeX, podendo ser adicionados "pacotes" que acrescentam inúmeras funcionalidades. É possível, portanto, o uso de classes ou pacotes para recriar a profusão de práticas idiossincráticas originadas de softwares de processamento de texto "visuais" como Word e LibreOffice, mas tal atitude é desencorajada porque não raramente obtêm-se resultados de qualidade nitidamente inferior, principalmente no que se refere à legibilidade e à harmonia e proporção do texto. ${ }^{8}$ Assim, pode-se afirmar que:

\begin{abstract}
A prática de fazer as coisas por si próprio já se difundiu amplamente há bastante tempo, mas os resultados são frequentemente duvidosos porque os tipógrafos leigos não veem o que está errado e não conseguem perceber o que é importante. Assim, fica-se acostumado a uma tipografia pobre e incorreta. [...] Ora, pode-se objetar que a tipografia depende do gosto. Se isso dissesse respeito somente a decoração, talvez deixássemos o argumento passar; entretanto, uma vez que a tipografia diz respeito principalmente à informação, erros podem não somente irritar, como também até causar danos (WILLBERG; FORSSMAN, 2000, apud KOHM; MORASWKI, 2015, p. 48-49).
\end{abstract}

E ainda, nas palavras de Tschichold:

Bom gosto e tipografia perfeita são suprapessoais. [...] Numa obraprima tipográfica, a assinatura do artista é eliminada. $O$ que alguns podem elogiar como estilos pessoais são, na realidade, pequenas e

8 Entre as práticas mais comuns encontradas, e que devem ser evitadas, estão o uso indevido de fontes (escolha e mistura de famílias com ou sem serifas, uso de várias fontes, fontes impróprias para a finalidade pretendida, uso espalhafatoso de cores, emprego do grifado e/ou negrito para ênfase) e a disposição do texto na página, procurando obter um maior rendimento de caracteres por página. Os espaços em branco - a margem do texto, e os espaços verticais entre seus vários elementos - são frequentemente tratados com verdadeiro horror vacui, mormente tendo por base um argumento econômico. No entanto, uma análise simples dos resultados obtidos com o mesmo texto segundo regras "técnicas" (e.g., ABNT) e outros parâmetros (LaTeX) desmente o argumento da "economia de papel". 
vazias peculiaridades, frequentemente danosas, que se disfarçam de inovações. [...] Tipografia pessoal é tipografia deficiente. Só iniciantes e bobos insistirão em usá-la (TSCHICHOLD, 2007, p. 25-26).

Mesmo assim, o uso do LaTeX, embora não privilegie a personalização no uso diário e trivial, permite-a claramente. É o caso de decorações de capitulares, ou modificações de fonte, margem, paginação, cabeçalhos e outros elementos, que podem ser facilmente mudados pelo usuário. Na figura 3, vemos um exemplo de decoração da página de abertura de um capítulo.

Finalmente, é também apontada como impedimento a dificuldade de aprendizado. Muitas coisas não podem ser feitas através do uso de menus com o mouse, embora o uso de um editor de textos especializado possa oferecer várias facilidades nesse sentido. Consequentemente, o usuário tem que aprender e compreender os comandos utilizados para obter o efeito desejado. Além disso, o modo de trabalho com um software que não é "visual", em que o resultado não é visto imediatamente (em inglês, representado pelo acrônimo WYSIWYG, "What you see is what you get"), pode desencorajar usuários que não têm familiaridade com programação. No entanto, com pouca prática e alguma instrução, em alguns minutos pode-se obter um resultado bastante satisfatório. Os comandos primários são bastante intuitivos: \title \{\} , para título; $\backslash$ author \{\} , para autor; $\backslash \operatorname{section}\{\}$, para uma nova seção; \tableofcontents para sumário etc. A figura 4 mostra um exemplo de artigo básico em LaTeX - o arquivo original e o arquivo em formato .pdf para visualização e impressão. O autor não precisa de se preocupar com formatação alguma: tudo já está definido pela "classe" - no caso, um "artigo" (article). Se um usuário iniciante desejasse escrever um artigo simples, que contém somente texto, isso é tudo o que precisaria saber, além de marcar a separação dos parágrafos com dois retornos (ENTER). Supondo que deseja enviar um artigo para o periódico Acta Mathematica, o autor deve enviar um arquivo .pdf gerado a partir de um documento LaTeX. Pouca ou nenhuma formatação é necessária. ${ }^{9}$

9 Ver instruções em: http://www.mittag-leffler.se/sites/default/files/documents/actaauthorinst.pdf. 
A utilização do sistema de preparação de textos LaTeX na produção de textos acadêmicos no Brasil: uma investigação preliminar e perspectivas

Figura 3 - Um exemplo de decorações feitas em LaTeX.

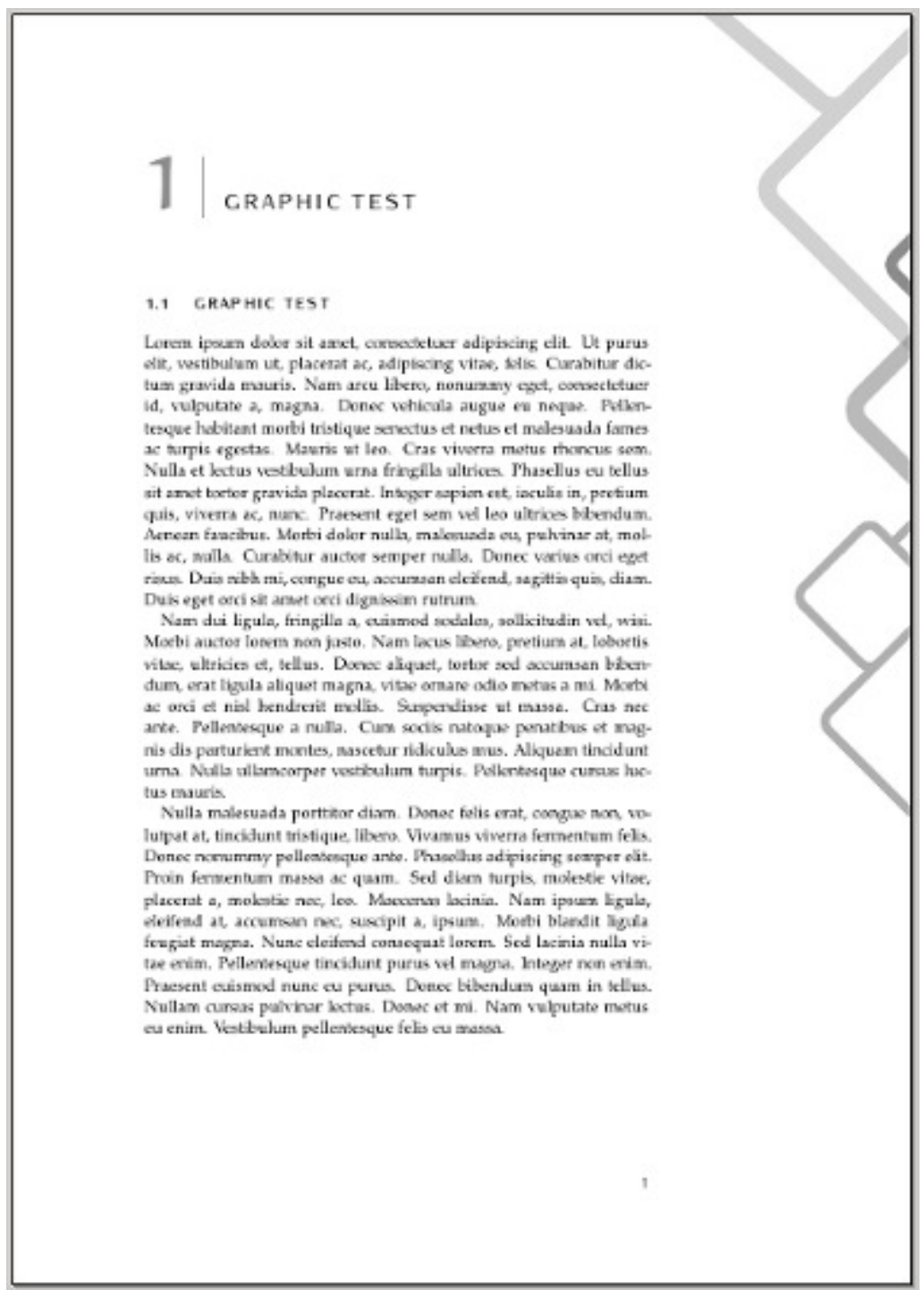

Fonte: Medina (2014). 
Youssef Alvarenga Cherem

A utilização do sistema de preparação de textos LaTeX na produção de textos acadêmicos no Brasil: uma investigação preliminar e perspectivas

Figura 4 - À esquerda: um exemplo de artigo em formato LaTeX (.tex). Abaixo, o resultado em formato.pdf.

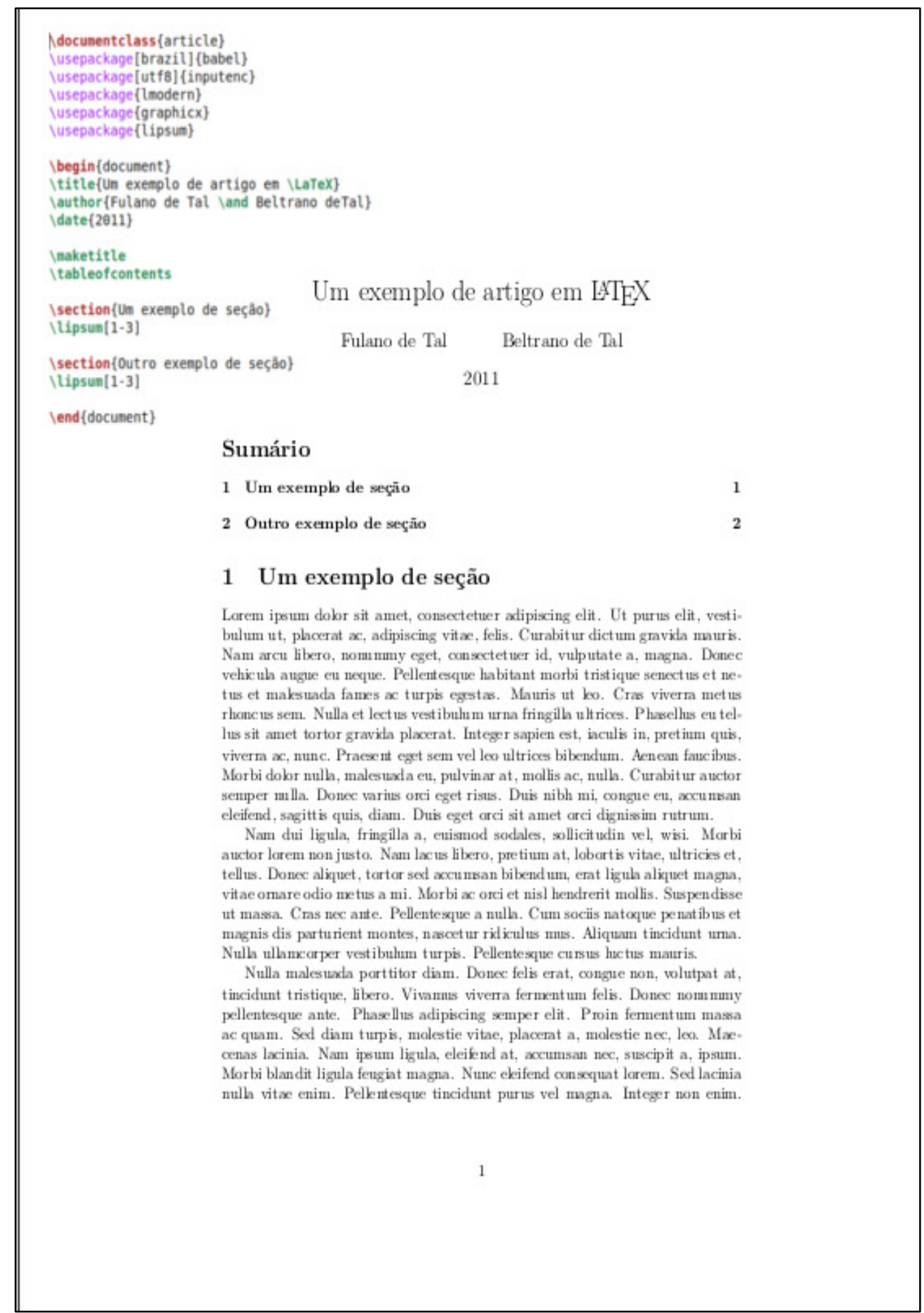

Fonte: $\mathrm{O}$ autor.

4 O USO DE LATEX NA ÁREA ACADÊMICA NO BRASIL: EXPERIÊNCIAS E DESAFIOS

Há vários anos o LaTeX tem sido utilizado no Brasil para a escrita e

Inf. Inf., Londrina, v. 20, n. 1, p. 228 - 249, jan./abr. 2015. 
A utilização do sistema de preparação de textos LaTeX na produção de textos acadêmicos no Brasil: uma investigação preliminar e perspectivas

divulgação de textos científicos de várias áreas. No entanto, a expansão de sua utilização tem encontrado vários obstáculos. O primeiro é o caso do relativo isolamento da produção científica naciona $\left.\right|^{10}$, o que implica também em uma defasagem relativa entre os desenvolvimentos de tecnologia da informação, da divulgação e difusão eletrônica da produção científica. Aqui não estamos afirmando que o mero uso de uma ferramenta de tipografia eletrônica contribuiria para a melhoria da qualidade da produção científica nacional, mas simplesmente que sua baixa utilização, no Brasil, em áreas onde essa ferramenta é prioritariamente utilizada (matemática, física, engenharia e ciência da computação), está em descompasso com seu uso amplamente difundido nessas áreas no exterior. Por outro lado, também não podemos deixar de ter em vista um amplo espectro de possibilidades, não só em toda a área acadêmica, inclusive as ciências humanas, mas também na editoração comercial. Um caso a ser notado é o da editora brasileira Hedra, que publica literatura utilizando LaTeX.

O desconhecimento e o baixo nível de emprego institucional e individual se reflete na paucidade e baixa qualidade de grande parte do material didático para esse software produzido em língua portuguesa. São vários os exemplos de erros conceituais e didáticos nas apostilas e introduções disponíveis - todas de forma eletrônica e gratuita - em português. Não obstante, existem vários projetos de desenvolvimento e divulgação do LaTeX no Brasil. Dentre eles se destaca o projeto abntex2. Arquitetado por Lauro César Araújo e mantido com a ajuda de uma comunidade de usuários, e cotando com o apoio do Centro de Pesquisa em Arquitetura da Informação (CPAl), da Universidade de Brasília, esse projeto de código livre retoma os propósitos de um projeto anterior, chamado abntex, cuja última versão estável é de 2004. Iniciado em 2012, o propósito do projeto abntex2 é criar uma classe LaTeX que contemple o cumprimento das normas da ABNT para trabalhos acadêmicos, inclusive os estilos bibliográficos. Nesse sentido a criação de uma classe LaTeX única que dê conta de todas as regras da ABNT já representa um grande desafio.

O principal uso das regras da ABNT se refere a trabalhos acadêmicos - a

10 Segundo Packer (2011), em 2009, 85\% dos artigos brasileiros indexados no Web of Science tiveram autores brasileiros exclusivamente. Ver também: Regalado (2010), Ladle, Todd e Machado (2012) e Righetti (2013). 
A utilização do sistema de preparação de textos LaTeX na produção de textos acadêmicos no Brasil: uma investigação preliminar e perspectivas

ABNT NBR 14724:2011 e suas "auxiliares". ${ }^{11}$ A maioria das universidades brasileiras exige, via de regra, o cumprimento de uma interpretação dessa norma para monografias, teses, dissertações e até trabalhos de disciplinas, portanto, existe um grande público em potencial. $O$ abntex2 automatiza quase toda a formatação dos trabalhos baseados nas normas da ABNT, bastando ao usuário utilizar as macros disponíveis - para título, capítulo, autor, instituição etc. - e os estilos bibliográficos ${ }^{12}$. O empecilho à criação e manutenção de um conjunto de macros tipográficas dessa natureza é que, por um lado, as normas da ABNT mudam com frequência, e, por outro, cada instituição, com sua interpretação própria das normas, pode exigir uma adaptação do estilo original $;{ }^{13}$ por conseguinte, a classe abntex 2 tem-se esforçado para ser flexível o bastante. Sua aceitação tem sido positiva; segundo o site de hospedagem do projeto (http://code.google.com/p/abntex2/) sua última versão teve 5.380 downloads - uma média de 1.300 downloads por mês.

Apesar disso, os usuários de LaTeX e da classe abntex2 muitas vezes esbarram em políticas burocráticas, vindas da parte de vários agentes acadêmicos orientadores, professores, comitês, secretários e bibliotecários. Existe pouco incentivo, e mesmo conhecimento, para a implementação de alternativas ao uso de processadores de texto como Microsoft Word e LibreOffice. Um dos maiores desafios, então, é conseguir apoio institucional oficial na área acadêmica e científica, de forma a permitir e fomentar seu uso, principalmente (mas não somente) nas áreas em que ele traz mais benefícios.

Neste artigo, apresentamos uma solução economicamente viável e profissional do ponto de vista dos resultados para a editoração de documentos, através do emprego do software LaTeX. Foram apresentados benefícios do ponto de vista do usuário ou redator do texto, bem como em relação a questões tipográficas como legibilidade e organização do texto impresso. O uso do LaTeX também é atrativo em relação ao custo-benefício. Apesar de ainda contar com uma base de usuários pequena no Brasil, o LaTeX e sua implementação nacional para as normas da ABNT, o projeto abntex2, têm um número crescente de usuários, atraídos por

11 ABNT NBR 6023:2002: Referências; ABNT NBR 6024:2012: numeração das seções de um documento; ABNT NBR 6027:2012: Sumário; ABNT NBR 6028:2003: Resumo; ABNT NBR 6034:2004: Índice; ABNT NBR 10520:2002: Citações.

${ }^{12}$ Ver documentação em Araújo (2014a, 2014b).

${ }^{13}$ Exemplo notório da falta de conhecimento das instituições acadêmicas sobre as normas da ABNT que supostamente seriam seguidas é a citação numérica, autorizada pela norma NBR 10520:2002, mas que mesmo assim frequentemente não são aceitas. 
uma opção de editoração eletrônica (desktop publishing) profissional, eficiente, estável e robusta.

\section{$5 \quad$ CONCLUSÕES}

A utilização de uma linguagem de mark-up como LaTeX pode trazer benefícios tanto do ponto de vista do produto final e sua relação com o leitor, quanto do ponto de vista do processo de edição do texto. Em relação ao produto final, LaTeX é um sistema tipográfico que proporciona um resultado satisfatório do ponto de vista estético, ajudando o leitor a visualizar dados, a realizar tanto uma leitura preliminar quanto profunda, a percorrer o texto e a encontrar informação. Do ponto de vista da redação e edição do texto, o LaTeX facilita o trabalho do autor ou redator para estruturar, dispor e apresentar a informação de modo a facilitar a leitura. Apesar disso, seu uso no Brasil é bastante restrito, mesmo em áreas em que seu benefício é mais tangível, como nas ciências exatas ou engenharias. No entanto, um dos maiores desafios para a disseminação de seu uso é a falta de apoio institucional oficial na área acadêmica e científica. Um primeiro passo para isso seria a aceitação desse sistema como ferramenta de trabalho, bem como uma divulgação mais ativa através do desenvolvimento de material didático e iniciativas de capacitação.

\section{AGRADECIMENTOS}

Lauro César Araújo e Paulo Roberto Massa Cereda.

\section{REFERÊNCIAS}

ARAÚJO, Lauro César. A classe abntex2: documentos técnicos e científicos brasileiros compatíveis com as normas ABNT. Jan. 2014a. Disponível em: <http://ctan.tche.br/macros/latex/contrib/abntex2/doc/abntex2.pdf>. Acesso em: 11 abr. 2014.

O pacote abntex2cite: estilos bibliográficos compatíveis com a ABNT NBR 6023. 2014b. Disponível em: <http://abntex2.googlecode.com/>. Acesso em: 11 abr. 2014. 
A utilização do sistema de preparação de textos LaTeX na produção de textos acadêmicos no Brasil: uma investigação preliminar e perspectivas

BAIN, Peter; SHAW, Paul (Ed.). Blackletter: type and national identity. Nova lorque: The Cooper Union for the Advancement of Science and Art and Princeton Architectural Press, 1998.

BLAIR, Sheila. Islamic calligraphy. Edinburgh: Edinburgh University Press, 2006.

BRASIL. Ministério do Planejamento. Guia livre: referência de migração para software livre do Governo Federa. Disponível em:

<http://www.governoeletronico.gov.br/ anexos/guia-livre-versao-1.0>. Acesso em: 16 abr. 2014.

BRINGHURST, Robert. The elements of typographic style: version 4.0: 20th anniversary edition. $4^{\text {th }}$ ed. Vancouver: Hartley and Marks, 2013.

CHAPPELL, Warren. A short history of the printed word. New York: Alfred A. Knopf, 1970.

CHEREM, Youssef. Uma introdução ao LaTeX: um sistema de preparação de textos. 2013. Mimeo.

DODD, Robin. From Gutenberg to opentype: an illustrated history of type from the earliest letterforms to the latest digital fonts. Vancouver: Hartley and Marks Publishers, 2006.

FELICI, James. The complete manual of typography. Berkeley: Peachpit Press, 2012.

GOOSENS, Michel; SAMARIM, Alexander; MITTELBACH, Frank; The LaTeX companion. Indianápolis: Addison-Wesley, 1994.

GRATZER, George A. Math into LaTeX. Boston; New York; Birkhäuser: Springer, 2000.

GUMBERT, J. Peter. Typography in the manuscript book. Journal of the Printing Historical Society, London, v. 22, p. 5-28, 1993.

INTERNATIONAL INSTITUTE FOR INFORMATION DESIGN (IIID). What is Information Design. Disponível em: <http://www.iiid.net/Information.aspx>. Acesso em: 18 de maio de 2014.

KING, Donald W. The cost of journal publishing: a literature review and commentary. Learned Publishing, Cambridgeshire, v. 20, n. 2, p. 85, 2007.

KOHM, Markus; MORAWSKI, Jens-Uwe. The KOMA-Script guide. Disponível em: http://texdoc.net/texmf-dist/doc/latex/koma-script/scrguien.pdf. 2015. Acesso em: 26 de junho de 2015.

KNUTH, Donald Ervin. The TeXBook. Reading: Addison-Wesley, 1984.

KOTTWITZ, Stefan. LaTeX Beginner's guide. Birmingham e Mumbai: Packt Publishing, 2011. 
A utilização do sistema de preparação de textos LaTeX na produção de textos acadêmicos no Brasil: uma investigação preliminar e perspectivas

LADLE, R. J.; TODD, Peter A.; MALHADO, Ana C. M. Assessing insularity in global science. Scientometrics, Amsterdam, n. 93, p. 745-750, 2012.

LAMPORT, Leslie. Latex: a document preparation system: user's guide and reference manual. Reading: Addison-Wesley, 1994.

LEHMAN, Philipp; KIME, Philip Kime; BORUVKA, Audrey Boruvka; WRIGHT, Joseph. The BibLaTeX package. Version 2.8a. 25/11/2013. Disponível em: $<$ http://mirrors.ctan.org/macros/latex/contrib/biblatex/doc/biblatex.pdf>. Acesso em: 16 abr. 2014.

LUPTON, Ellen (2010). Thinking with Type. New York: Princeton Architectural Press, 2004.

MEDINA, Gonzalo. Trying to do graphical decorations in "ClassicThesis style". Disponível em: <http://tex.stackexchange.com/questions/86294/ trying-to-dographical-decorations-in-classicthesis-style/86310\#86310 >. Acesso em: 14 abr. 2014.

OETIKER, Tobias; PARTL, Hubert; HYNA, Irene; SCHLEGL, Elisabeth. The Not So Short Introduction to LaTeX2ع. Version 5.01, April 06, 2011. Disponível em: <http://mirrors.ctan.org/info/lshort/english/lshort.pdf>. Acesso em: 16 abr. 2014.

PACKER, Abel L. Os periódicos brasileiros e a comunicação da pesquisa nacional. Revista USP, São Paulo, n. 89, maio 2011. Disponível em: $<$ http://rusp.scielo.br/scielo.php? script=sci_arttext\&pid=S010399892011000200004\&Ing=pt\&nrm=iso >. Acesso em: 7 jun. 2014.

PETTERSSON, R. It Depends: ID - principles and guidelines. $4^{\text {th }}$ ed. Tullinge: IIID, 2012. Disponível em: <http://www.iiid.net/PDFs/ltDepends.pdf>. Acesso em: 7 jun. 2014

POHLEN, Joep; SETOLA, Geert. Letter fountain. Colonia: Taschen, 2011.

POOLE, Alex. Which are more legible: serif or sans serif typefaces? 2008.

Disponível em: <http://alexpoole.info/blog/which-are-more-legible-serif-or-sans-seriftypefaces/>. Acesso em: 11 abr. 2014.

PUNCTUATION. In: BROWN, T. Julian. Encyclopaedia britannica, 2011. Disponível em: <http://www.britannica.com/EBchecked/topic/483473/punctuation>. Acesso em: 11 abr. 2014.

REGALADO, Antonio. Brazilian Science: Riding a Gusher. Science, 3 December 2010, Vol. 330, no. 6009, pp. 1306-1312.

RIGHETTI, Sabine. Produção científica do Brasil aumenta, mas qualidade cai. Folha De São Paulo, São Paulo, 22 abr. 2013. Disponível em: <http://www1.folha.uol.com.br/fsp/cienciasaude/105099-producao-cientifica-do-brasilaumenta-mas-qualidade-cai.shtml>. Acesso em: 7 jun. 2014.

RUDER, Emil. Typographie. 3. ed. Niederteufen: Arthur Niggli, 1977. 
A utilização do sistema de preparação de textos LaTeX na produção de textos acadêmicos no Brasil: uma investigação preliminar e perspectivas

SAENGER, Paul. Space between words: the origins of silent reading. Stanford: Stanford University Press, 2000.

SOCIEDADE BRASILEIRA DE DESIGN DA INFORMAÇÃO - SBDI. Disponível em: <http://www.sbdi.org.br/>. Acesso em: 14 abr. 2014.

SOCIETY FOR TECHNICAL COMMUNICATION. Information Design Special Interest Group. Definitions of information design. 2005. Disponível em: <http://www.stcsig.org/id/id_definitions.htm>. Acesso em: 18 maio 2014.

THE FREE SOFTWARE FOUNDATION. What is free software? Disponível em: <https://www.gnu.org/philosophy/free-sw.html . Acesso em: 14 abr. 2014.

THE OPEN SOURCE INITIATIVE. The open source definition. Disponível em: <http://opensource.org/docs/osd>. Acesso em: 14 abr. 2014.

TRACY, Walter. Letters of credit. A view of type design. Boston: David R. Godine, 2003.

TSCHICHOLD, Jan. A forma do livro: ensaios sobre tipografia e estética do livro. São Paulo: Ateliê, 2007.

UNGER, Gerard. Typografie als voertuig van de wetenschap: typography as a vehicle of science. Leiden: Uitgeverij De Buitenkant, 2007.

Van DONGEN, Marc R. C. Latex and friends. Berlin and Heidelberg: SpringerVerlag, 2012.

WILLBERG, Hans Peter; FORSSMAN, Friedrich. Erste hilfe in typografie. Mainz: Verlag Hermann Schmidt, 2000.

\section{Title}

Usage of LaTeX for typesetting scholarly texts in Brazil: a preliminary study and future perspectives

\section{Abstract}

Introduction: LaTeX is a document preparation system based on the TeX typesetting system. We point out the main benefits and challenges in using this tool, as well as usage perspectives in the short and medium terms in Brazil.

Objective: The objective of this article is to analyze the usage of the LaTeX typesetting system in the production of scholarly texts in Brazil.

Methodology: Literature review and document research.

Results: The usage of a mark-up language like LaTeX can bring benefits from the point of view of the final product and its relation with the reader as well as from the point of view of the process of editing the text. However, its usage in Brazil is extremely limited.

Conclusion: We argue that one of the main challenges for disseminating its usage is the 
A utilização do sistema de preparação de textos LaTeX na produção de textos acadêmicos no Brasil: uma investigação preliminar e perspectivas

lack of institutional support in the scientific and academic communities. Its acceptance by institutions, as well as initiatives for disseminating technical skills, are pointed as factors that can change this situation.

Keywords: Graphic design. Typography. Information design. Scientific communication.

\section{Título}

Utilización del sistema para preparación de documentos LaTeX en la producción de documentos académicos en Brasil: una investigación preliminar y perspectivas

\section{Resumen}

Introducción: LaTeX es un sistema de composición de textos desarrollado para el sistema tipográfico TeX. En este artículo, presentamos los principales beneficios y desafíos de la utilización de esta herramienta, así como las perspectivas para su uso en Brasil en el corto y mediano plazo.

Objetivo: Analizar el uso de software LaTeX en la producción de textos académicos en Brasil.

Metodología: Revisión de la literatura y investigación documental.

Resultados: El uso de un lenguaje de marcado como LaTeX puede beneficiar tanto el producto final y su relación con el lector, cuanto el proceso de edición de texto. Sin embargo, su uso en Brasil es muy limitado.

Conclusiónes: Se argumenta que uno de los mayores desafíos para la propagación de su uso es la falta de apoyo oficial institucional en el ámbito académico y científico. Su aceptación institucional, así como iniciativas de difusión de conocimientos técnicos, son señalados como factores que pueden cambiar esta situación.

Palabras-clave: Diseño gráfico. Tipografía. Diseño de la información. Comunicación científica.

Recebido em: 10.06.2014

Aceito em: 07.06.2015 